\title{
Clinical Outcomes of First-line Sunitinib Followed by Immuno-oncology Checkpoint Inhibitors in Patients With Metastatic Renal Cell Carcinoma
}

\author{
J. Connor Wells, ${ }^{1}$ Jeffrey Graham, ${ }^{2}$ Benoit Beuselinck, ${ }^{3}$ Georg A. Bjarnason, ${ }^{4}$ \\ Frede Donskov, ${ }^{5}$ Aaron R. Hansen, ${ }^{6}$ Rana R. McKay, ${ }^{7}$ Ulka Vaishampayan, ${ }^{8}$ \\ Guillermo De Velasco, ${ }^{9}$ Mei S. Duh, ${ }^{10}$ Lynn Huynh, ${ }^{10}$ Catherine Nguyen, ${ }^{10}$ \\ Giovanni Zanotti, ${ }^{11}$ Krishnan Ramaswamy, ${ }^{11}$ Toni K. Choueiri, \\ Daniel Y.C. Heng ${ }^{1}$
}

Abstract

Limited data are available on how the first-line duration can affect clinical outcomes observed after subsequent immuno-oncology treatment among patients with metastatic renal cell carcinoma. The present retrospective cohort study of 161 patients found that the first-line sunitinib duration might have a minimal effect on subsequent immuno-oncology therapy effectiveness. These findings could aid healthcare practitioners in the treatment decision process for sequencing therapies.

Background: The present retrospective, longitudinal cohort study assessed the association between the firstline sunitinib treatment duration and clinical outcomes with second-line immuno-oncology (IO) therapy among patients with metastatic renal cell carcinoma (mRCC). Patients and Methods: A total of 161 patients with mRCC who had been treated with first-line sunitinib and subsequent IO therapy from select International mRCC Database Consortium centers were included. The overall survival, time to next therapy, time to treatment discontinuation, and real-world physician-assessed best response measured from 10 therapy initiation were analyzed and compared between patients treated with first-line sunitinib for $\geq 6$ months and those treated for $<6$ months. Results: The 116 patients treated with sunitinib for $\geq 6$ months tended to be older and to have a better International mRCC Database Consortium risk than the 45 patients treated for $<6$ months (favorable, $36 \%$ vs. $8 \%, P=$ .001 ; intermediate, $59 \%$ vs. $70 \%, P=.21$; poor, $5 \%$ vs. $22 \%, P=.007$ ). The receipt of sunitinib for $\geq 6$ months versus $<6$ months was associated with longer survival (hazard ratio [HR], 0.42; 95\% confidence interval [Cl], 0.21 $0.87 ; P=.02)$. No significant association was observed between the first-line sunitinib duration and second-line 10 outcomes, including the time to next therapy $(\mathrm{HR}, 0.89 ; 95 \% \mathrm{Cl}, 0.52-1.51 ; P=.66)$, time to treatment discontinuation (HR, 0.85; 95\% Cl, 0.54-1.34; $P=.49$ ), and tumor response (odds ratio, $0.73 ; 95 \% \mathrm{Cl}, 0.22-2.49 ; P=.62$ ). Conclusions: We found no statistically significant association between the first-line sunitinib duration and clinical outcomes with second-line IO therapy. Patients receiving first-line sunitinib for $\geq 6$ months compared with $<6$ months was associated with better overall survival, although potential unadjusted confounders could have been

\footnotetext{
${ }^{1}$ Department of Oncology, University of Calgary, Calgary, AB, Canada

${ }^{2}$ Department of Oncology, CancerCare Manitoba, University of Manitoba, Winnipeg,

$\mathrm{MB}$, Canada

${ }^{3}$ Department of Oncology, University Hospital Leuven, Kathoieke Universiteit

Leuven, Leuven, Belgium

${ }^{4}$ Department of Oncology, Sunnybrook Health Sciences Centre, Toronto, ON,

Canada

${ }^{5}$ Department of Oncology, Aarhus University Hospital, Aarhus, Denmark

${ }^{6}$ Department of Oncology, Princess Margaret Cancer Centre, Toronto, ON, Canada

${ }^{7}$ Department of Oncology, University of California, San Diego, San Diego, CA

${ }^{8}$ Department of Oncology, Karmanos Cancer Institute, Detroit, MI
}

${ }_{9}^{9}$ Department of Oncology, University Hospital 12 de Octubre, Madrid, Spain
${ }^{10}$ Analysis Group, Inc, Boston, MA
${ }^{11}$ Pfizer, Inc, New York, NY
${ }^{12}$ Department of Oncology, Dana-Farber Cancer Institute, Boston, MA

Submitted: Oct 25, 2019; Revised: Dec 2, 2019; Accepted: Dec 9, 2019

Address for correspondence: Daniel Y. C. Heng, MD, MPH, FRCPC, University of Calgary, Calgary, AB, Canada

E-mail contact: Daniel.Heng@albertahealthservices.ca 


\section{Sunitinib Followed by IO Checkpoint Inhibitors for mRCC}

present. These findings support the paradigm that previous therapy will not dictate the effectiveness of subsequent immunotherapy.

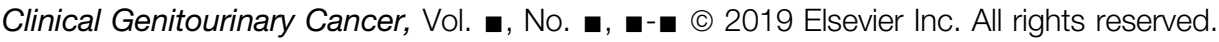

Keywords: $\mathrm{mRCC}$, Real-world effectiveness, Subsequent treatment, Targeted therapy, Treatment outcomes

\section{Introduction}

Immuno-oncology (IO) therapies have revolutionized the treatment paradigm for metastatic renal cell carcinoma (mRCC), adding to standard therapies, including tyrosine kinase inhibitors (TKIs), antivascular endothelial growth factor (VEGF) antibodies, and mammalian target of rapamycin inhibitors. ${ }^{1,2}$ IO therapy includes immune checkpoint inhibitors such as nivolumab, which was approved in 2015 for treatment of advanced RCC after previous antiangiogenic therapy. ${ }^{3,4}$ In 2018, combination nivolumab plus ipilimumab was approved for patients with treatment-naive International Metastatic Renal Cell Carcinoma (mRCC) Database Consortium (IMDC) intermediate- or poor-risk advanced RCC.,6 Additional first-line IO therapies combined with VEGF inhibitor therapies, including avelumab plus axitinib and pembrolizumab plus axitinib, were evaluated in clinical trials and approved in 2019. ${ }^{7,8}$

Limited data are available on how the first-line duration and clinical response might affect the clinical outcomes observed immediately after subsequent IO treatment. Previous studies have shown that sunitinib inhibits angiogenesis, has direct antitumor effects, and exacerbates intratumoral heterogeneity. Also, IO therapies might be more effective for tumors with a high mutational burden. ${ }^{9-11}$ A subgroup analysis of the phase III CheckMate 025 trial demonstrated that patients with $\geq 6$ months of first-line therapy with sunitinib, pazopanib, or interleukin-2 versus those with $<6$ months had better median overall survival (OS) when subsequently treated with nivolumab (27.4 vs. 18.2 months) or everolimus (22.8 vs. 14.0 months). ${ }^{12}$ Further study of the effect of first-line treatment duration on the second-line clinical outcomes is warranted, given the changing treatment landscape.

The objective of the present study was to evaluate the effect of the first-line sunitinib treatment duration $(\geq 6$ months vs. $<6$ months) before IO therapy on the clinical outcomes in a real-world setting using data from the IMDC.

\section{Patients and Methods Study Design and Study Population}

The present retrospective, longitudinal cohort study used data from the patient medical records at select IMDC cancer centers in Belgium, Canada, Denmark, and the United States. Uniform database templates and standardized definitions were used to ensure the data were collected consistently. ${ }^{13}$ The eligible patients were adults with mRCC who had initiated first-line sunitinib therapy from 2006 to 2018 and had received subsequent IO therapy, either alone or combined with another therapy.

The observation period spanned the date from IO therapy initiation (index date) to the date of last contact or death. The baseline period was defined as the interval from the mRCC diagnosis to the index date. Data on the demographic and clinical characteristics during the baseline period to the index date were collected. The sunitinib treatment duration was defined as the interval from treatment initiation to discontinuation for any reason. The IMDC prognostic risk group was computed at sunitinib initiation and the index date according to the individual risk factors (ie, $<1$ year from RCC diagnosis to sunitinib initiation, Karnofsky performance status $<80 \%$, serum hemoglobin less than the lower limit of normal, corrected calcium greater than the upper limit of normal $[\mathrm{ULN}]$, neutrophil count greater than the ULN, platelet count greater than the ULN). Those with no risk factors were deemed to have favorable risk, those with 1 to 2 risk factors were deemed to have intermediate risk, and those with $\geq 3$ factors were deemed to have poor risk. ${ }^{13}$

Data on the clinical characteristics, treatment patterns, and clinical endpoints in the follow-up period were collected. OS for IO therapy was defined as the interval from IO therapy initiation to death. The time to next therapy (TTNT) was defined as the interval from IO therapy initiation to initiation of the next line of therapy or death. The TTNT was used as an objective proxy for progressionfree survival (PFS) owing to the difficulty in determining the true timing of progression during IO therapy, with many patients deriving clinical benefit from IO therapy even after discontinuing because of adverse events. The time to treatment discontinuation (TTD) for IO therapy was defined as the interval from IO therapy initiation to IO therapy discontinuation for any reason. The reasons for treatment discontinuation were collected. The real-world physician-assessed best response was determined from clinical and/ or radiographic criteria using the Response Evaluation Criteria in Solid Tumors guidelines, with the imaging assessments occurring at clinically variable intervals. The objective response rate (ORR) was reported as the proportion of patients with a partial or complete response. $^{14}$

All data were de-identified and complied with the patient confidentiality requirements of the Health Insurance Portability and Accountability Act. The local institutional review board at each of the institutions approved all the study materials.

\section{Statistical Analysis}

The patients were categorized into treatment groups according to the duration of first-line sunitinib therapy. A 6-month threshold was chosen to reflect previous studies, which had investigated $\geq 6$ months and $<6$ months. ${ }^{12,15}$ Descriptive statistics were calculated using frequencies and proportions for categorical variables and the mean \pm standard deviation, and median for continuous variables. Differences between patients were compared using the Pearson $\chi^{2}$ test or the Fisher exact test, as appropriate, for categorical variables, and continuous variables were compared using $t$ tests or Wilcoxon rank-sum tests.

The real-world treatment patterns were described using a flow chart of the time between treatments using the median and 
Table 1 Baseline Demographic and Clinical Characteristics

\begin{tabular}{|c|c|c|c|}
\hline \multirow[b]{2}{*}{ Characteristic } & \multicolumn{2}{|c|}{ First-line Sunitinib Duration } & \multirow[b]{2}{*}{$P$ Value } \\
\hline & $<6 \mathrm{mo}(\mathrm{n}=45)$ & $\geq 6 \mathrm{mo}(\mathrm{n}=116)$ & \\
\hline \multicolumn{4}{|l|}{ Demographic } \\
\hline Age at index date, $y$ & & & $.004^{\mathrm{b}}$ \\
\hline Mean \pm SD & $57.8 \pm 10.7$ & $63.4 \pm 11.1$ & \\
\hline Median & 58.9 & 65.0 & \\
\hline Race, n (\%) & & & .85 \\
\hline White & $25(75.8)$ & $60(74.1)$ & \\
\hline Nonwhite & $8(24.2)$ & $21(25.9)$ & \\
\hline Sex, n (\%) & & & .98 \\
\hline Male & $35(77.8)$ & $90(77.6)$ & \\
\hline Female & $10(22.2)$ & $26(22.4)$ & \\
\hline \multicolumn{4}{|l|}{ Tumor characteristic } \\
\hline Pathologic findings, n (\%) & & & .08 \\
\hline Clear cell & $32(76.2)$ & 98 (87.5) & \\
\hline Nonclear cell & $10(23.8)$ & $14(12.5)$ & \\
\hline Sarcomatoid features, n (\%) & & & .54 \\
\hline Yes & $3(9.7)$ & $12(16.2)$ & \\
\hline No & $28(90.3)$ & $62(83.8)$ & \\
\hline No. of metastases, $n(\%)$ & & & .88 \\
\hline 1 & $8(19.5)$ & $21(20.6)$ & \\
\hline$>1$ & $33(80.5)$ & $81(79.4)$ & \\
\hline Brain metastases, n (\%) & & & .67 \\
\hline Yes & $1(3.3)$ & $5(7.0)$ & \\
\hline No & $29(96.7)$ & $66(93.0)$ & \\
\hline \multicolumn{4}{|l|}{ Previous treatment } \\
\hline Previous nephrectomy, n (\%) & & & $.0005^{\mathrm{b}}$ \\
\hline Yes & $32(71.1)$ & $107(92.2)$ & \\
\hline No & $13(28.9)$ & $9(7.8)$ & \\
\hline Previous IL-2 or IFN therapy, n (\%) & & & .67 \\
\hline Yes & $1(2.2)$ & $6(5.2)$ & \\
\hline No & $44(97.8)$ & $110(94.8)$ & \\
\hline \multicolumn{4}{|l|}{ Year of sunitinib initiation, $\mathrm{n}(\%)$} \\
\hline $2006-2009$ & $1(2.2)$ & $4(3.4)$ & $>.99$ \\
\hline $2010-2013$ & 7 (15.6) & $34(29.3)$ & .07 \\
\hline $2014-2017$ & $37(82.2)$ & 78 (67.2) & .06 \\
\hline $\begin{array}{l}\text { IMDC prognosis risk group at first-line, } \\
\mathrm{n}(\%)\end{array}$ & 37 & 99 & \\
\hline Favorable & $3(8.1)$ & $36(36.4)$ & $.001^{\mathrm{b}}$ \\
\hline Intermediate & $26(70.3)$ & $58(58.6)$ & .21 \\
\hline Poor & $8(21.6)$ & $5(5.1)$ & $.007^{\mathrm{b}}$ \\
\hline $\begin{array}{l}\text { IMDC prognosis risk group at second- } \\
\text { line, } n(\%)\end{array}$ & 41 & 98 & \\
\hline Favorable & $2(4.9)$ & $12(12.2)$ & .23 \\
\hline Intermediate & $29(70.7)$ & $69(70.4)$ & .97 \\
\hline Poor & $10(24.4)$ & $17(17.4)$ & .34 \\
\hline
\end{tabular}

Abbreviations: IFN = interferon; IL-2 = interleukin-2; IMDC = International Metastatic Renal Cell Carcinoma Database Consortium; $10=$ immuno-oncology; SD = standard deviation

${ }^{\mathrm{a} F o r}$ categorical variables, proportions were calculated after excluding those patients with missing data.

${ }^{\mathrm{b}} \mathrm{P}<.05$. 


\section{Sunitinib Followed by IO Checkpoint Inhibitors for mRCC}

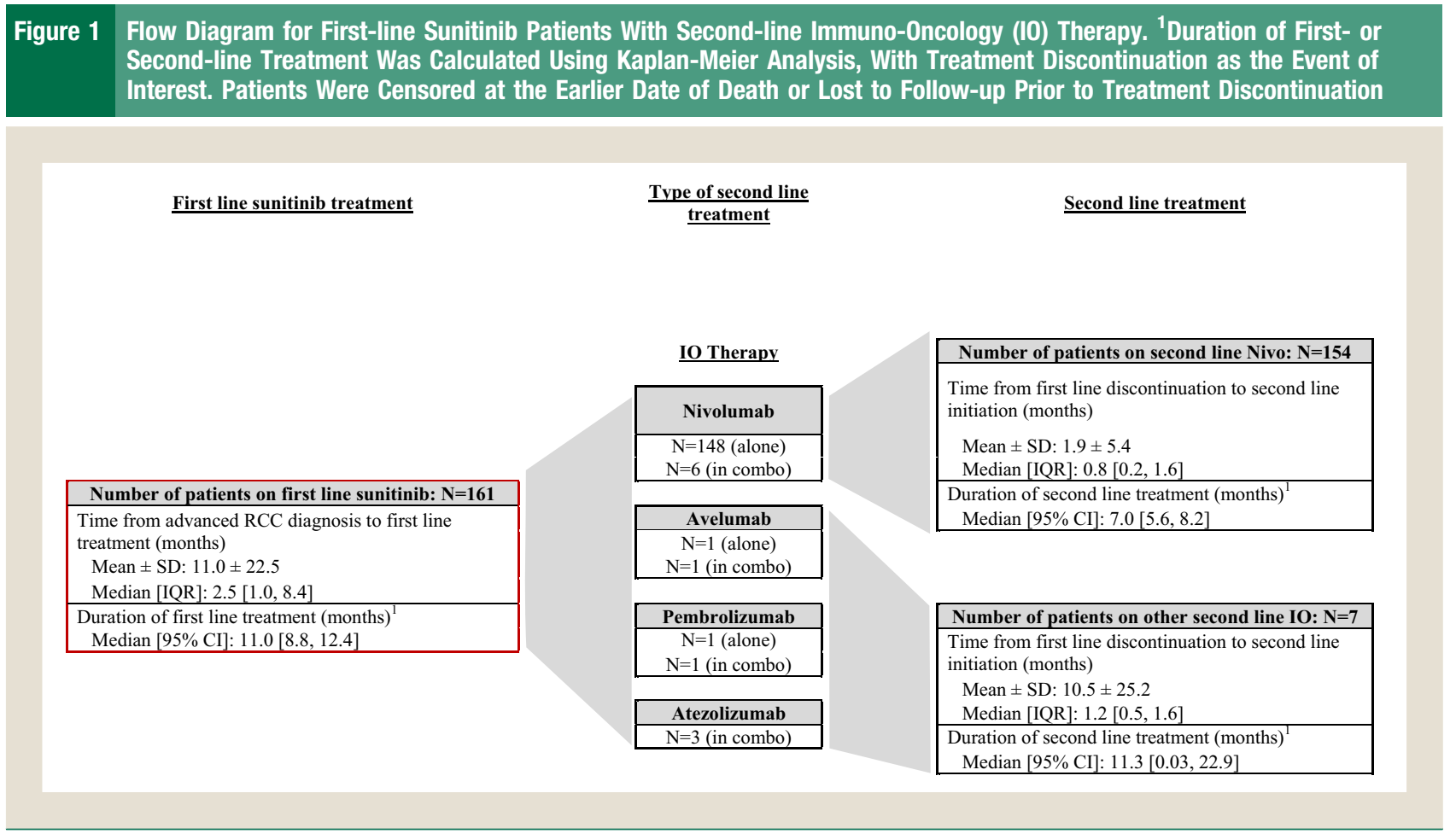

Abbreviations: $\mathrm{Cl}=$ confidence interval; $\mathrm{IQR}=$ interquartile range; Nivo = nivolumab; $\mathrm{RCC}=$ renal cell carcinoma; $\mathrm{SD}=$ standard deviation.

interquartile range and mean \pm standard deviation. The durations of first-line sunitinib and second-line IO therapy were calculated for each patient and plotted and compared using a scatter plot and the Pearson correlation coefficient. For the timeto-event analyses (ie, OS, TTNT, TTD), Kaplan-Meier analyses and Cox proportional hazards models were used to estimate the hazard ratios (HRs) and 95\% confidence intervals (CIs). For the TTNT and TTD analyses, the models were adjusted for age, sex, and baseline IMDC risk group. For the OS analysis, inverse probability weighting (IPW)-adjusted estimates were calculated. ${ }^{16}$ The model included time-varying weights to predict the probability of censoring, discontinuing IO therapy, and initiating a third-line therapy because these could influence the OS and could have varied according to the duration of first-line sunitinib. The weights were estimated using data on the sunitinib duration, baseline IMDC risk group, age, sex, and IMDC risk group at the initiation of IO.

The real-world first-line sunitinib and second-line IO physicianassessed best tumor responses were compared between groups using the $\chi^{2}$ test for trend. In addition, the ORR for second-line IO therapy was assessed using multivariable logistic regression models. The odds ratios, with 95\% CIs, and $P$ values are reported.

All analyses were performed using SAS, version 9.4 (SAS Institute, Inc, Cary, NC).

\section{Results}

\section{Demographic and Clinical Characteristics}

Of the 161 patients with $\mathrm{mRCC}, 116$ had received $\geq 6$ months and 45 patients had received $<6$ months of first-line sunitinib treatment (Table 1). Overall, the median duration of first-line sunitinib was 11.0 months (Figure 1). A greater proportion of patients treated $\geq 6$ months versus $<6$ months with first-line sunitinib were older and had previously undergone nephrectomy (mean age at index date, 63 vs. 58 years, $P=.004$; previous nephrectomy, $92 \%$ vs. $71 \%, P<.001$ ). Patients with $\geq 6$ months of first-line sunitinib treatment also tended to have a better IMDC risk at the initiation of sunitinib compared with patients with $<6$ months of treatment. At the initiation of second-line IO, patients with $\geq 6$ months of first-line sunitinib had had an IMDC risk similar to that for those treated for $<6$ months. From the start of first-line sunitinib to the start of second-line IO, 74 patients (53\%) had remained within the same IMDC risk group, 7 patients $(5 \%)$ had moved to an improved IMDC group, and 41 patients (30\%) had moved to a worse IMDC risk group.

\section{Treatment Patterns}

Most patients ( $\mathrm{n}=154$; 96\%) had received nivolumab as their second-line IO therapy, although others had received avelumab, atezolizumab, or pembrolizumab as second-line IO therapy. A slightly greater proportion of patients with $\geq 6$ months versus $<6$ months of first-line sunitinib treatment had received third-line treatment (39\% vs. $33 \%$; $P=.52$; Supplemental Table 1; available in the online version). No significant association was observed between the first-line sunitinib duration and second-line IO duration on a scatterplot analysis among all patients (Pearson correlation coefficient, $0.040 ; P=.62$; Figure 2 ). For the patients who discontinued second-line IO therapy, we also found no significant association between the first-line sunitinib treatment duration and second-line IO therapy duration using a scatterplot analysis (Pearson correlation coefficient, $0.034 ; P=.73$ ). 
Figure 2 Scatterplot Analysis of Treatment Duration for First-line Sunitinib Versus Second-line Immune-oncology (I0) Therapy for All Patients

First-line sunitinib vs. second-line IO therapy $(\mathrm{N}=161)$

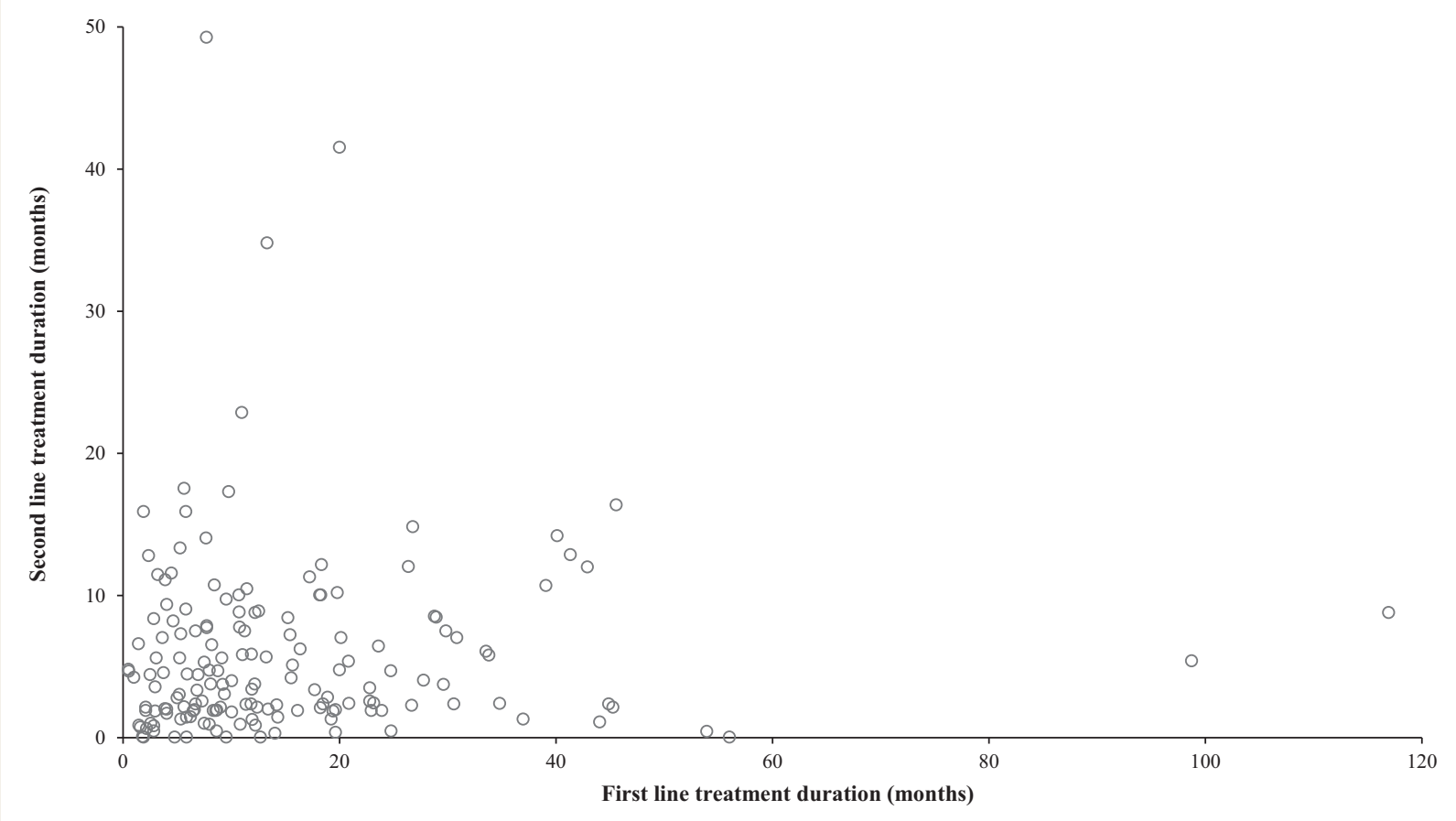

\section{Clinical Outcomes}

The unadjusted median OS was numerically longer for the patients treated with $\geq 6$ months of first-line sunitinib than for those treated with $<6$ months (median, 24.9 months; 95\% CI, 16.447.8 months; vs. median, 17.5 months; 95\% CI, 10.3-30.9 months). In the adjusted OS analyses using IPW, the patients treated with $\geq 6$ months of first-line sunitinib had a significantly lower hazard of death compared with the patients treated for $<6$ months (adjusted HR, 0.42; 95\% CI, 0.21-0.87; $P=.02$; Table 2). In the unadjusted analyses, the median TTNT was numerically greater for patients treated with $\geq 6$ months of first-line sunitinib compared with those treated for $<6$ months (median TTNT, 9.2 months; 95\% CI, 6.8-11.3 months; vs. median TTNT, 8.0; 95\% CI, 5.6-10.8 months). However, no significant association was observed in the adjusted analyses for TTNT (Table 2). The unadjusted median TTD was numerically greater for the patients receiving $\geq 6$ months of first-line sunitinib treatment group than for the patients receiving $<6$ months (median TTD, 7.0 months; 95\% CI, 4.7-8.4 months; vs. median TTD, 4.8 months; 95\% CI, 3.0-8.2 months). However, no significant association was observed in the adjusted analysis (Table 2).

The reasons for treatment discontinuation are presented in Table 3. Numerically fewer patients with $\geq 6$ months of sunitinib treatment compared with those receiving $<6$ months had

\section{Table 2 Analysis of Treatment Outcomes For Patients Who Had Received First-line Sunitinib Before Second-line 10 Therapy}

\begin{tabular}{|c|c|c|c|c|}
\hline \multirow[b]{2}{*}{ Variable } & \multicolumn{3}{|c|}{ HR (95\% CI) } & \multirow{2}{*}{$\begin{array}{c}\text { ORR } \\
\text { OR (95\% Cl) }\end{array}$} \\
\hline & OS & TTNT of IO Therapy & TTD for 10 Therapy & \\
\hline $\begin{array}{l}\text { Sunitinib duration } \geq 6 \mathrm{mo} \\
\text { (ref, }<6 \mathrm{mo} \text { ) }\end{array}$ & $0.42(0.21-0.87)$ & $0.89(0.52-1.51)$ & $0.85(0.54-1.34)$ & $0.73(0.22-2.49)$ \\
\hline $\begin{array}{l}\text { Favorable/intermediate IMDC } \\
\text { prognosis risk group (ref, poor } \\
\text { risk group) }\end{array}$ & $0.58(0.19-1.79)$ & $0.52(0.29-0.96)$ & $0.58(0.34-0.98)$ & $0.46(0.13-1.64)$ \\
\hline $\begin{array}{l}\text { Age } \geq 60 \text { y at index date (ref, } \\
\text { age }<60 \text { y) }\end{array}$ & $1.99(0.95-4.19)$ & $0.76(0.46-1.26)$ & $0.89(0.57-1.38)$ & $0.33(0.11-1.05)$ \\
\hline Sex (ref, female) & $1.64(0.66-4.12)$ & $0.88(0.48-1.61)$ & $0.78(0.47-1.28)$ & $2.23(0.44-11.28)$ \\
\hline
\end{tabular}

Abbreviations: $\mathrm{Cl}=$ confidence interval; $\mathrm{HR}=$ hazard ratio; IMDC = International Metastatic Renal Cell Carcinoma Database Consortium; IO = immuno-oncology; OR = odds ratio; ORR = overall response rate; ref $=$ reference; $T \mathrm{DD}=$ time to treatment discontinuation; TTNT $=$ time to next therapy. 


\section{Sunitinib Followed by IO Checkpoint Inhibitors for mRCC}

Table 3 Reasons for Treatment Discontinuation Stratified by First-line Sunitinib Duration

\begin{tabular}{|c|c|c|c|}
\hline \multirow[b]{2}{*}{ Characteristic } & \multicolumn{2}{|c|}{ First-line Sunitinib Duration } & \multirow[b]{2}{*}{$P$ Value } \\
\hline & $<6 \mathrm{mo}(\mathrm{n}=45)$ & $\geq 6 \mathrm{mo}(\mathrm{n}=116)$ & \\
\hline \multicolumn{4}{|l|}{ First-line treatment } \\
\hline Patients who discontinued, $\mathrm{n}$ & 45 & 116 & \\
\hline \multicolumn{4}{|l|}{$\begin{array}{l}\text { Reason for treatment discontinuation, } \\
\mathrm{n}(\%)\end{array}$} \\
\hline Disease progression & $28(84.8)$ & $73(76.0)$ & .29 \\
\hline Toxicity & $3(9.1)$ & $15(15.6)$ & .56 \\
\hline Disease progression and toxicity & $0(0.0)$ & $5(5.2)$ & .33 \\
\hline Other & $2(6.1)$ & $3(3.1)$ & .60 \\
\hline \multicolumn{4}{|l|}{ Second-line treatment ${ }^{\mathrm{a}}$} \\
\hline Patients who discontinued & 31 & 75 & \\
\hline \multicolumn{4}{|l|}{$\begin{array}{l}\text { Reason for treatment discontinuation, } \\
\mathrm{n}(\%)\end{array}$} \\
\hline Disease progression & $17(73.9)$ & $41(64.1)$ & .39 \\
\hline Toxicity & $2(8.7)$ & $12(18.8)$ & .34 \\
\hline Disease progression and toxicity & $0(0.0)$ & $2(3.1)$ & NA \\
\hline Death & $4(17.4)$ & $7(10.9)$ & .47 \\
\hline Other & $0(0.0)$ & $2(3.1)$ & NA \\
\hline
\end{tabular}

Abbreviations: $10=$ immuno-oncology; NA = not applicable.

ap $<.05$.

discontinued sunitinib because of disease progression (76\% vs. $85 \% ; P=.29)$ or had discontinued IO therapy because of disease progression (64\% vs. $74 \% ; P=.39)$.

Patients with $\geq 6$ months of first-line sunitinib versus $<6$ months had a significantly greater ORR (30\% vs. $6 \% ; P=.01)$ during sunitinib therapy. During second-line IO therapy, the ORR was similar for patients with $\geq 6$ months of first-line sunitinib versus $<6$ months $(18 \%$ vs. $23 \%, P=.61$; Supplemental Table 2; available in the online version). No significant association was observed between the first-line sunitinib tumor response and the second-line IO tumor response $(P=.48$; Figure 3$)$.

\section{Discussion}

In the present real-world analysis of patients with mRCC, the TTNT, TTD, and tumor response to second-line IO therapy were not significantly different between patients who had received $\geq 6$ months and those who had received $<6$ months of first-line sunitinib. These results suggest that first-line sunitinib therapy resistance does not preclude a second-line IO therapy response and that the mechanisms of resistance are likely independent. The present study also found that patients treated with first-line sunitinib for $\geq 6$ months versus $<6$ months had significantly better OS. Thus, the first-line sunitinib duration might serve as a disease prognosis marker. The results from the present study and others have provided information on how the initial targeted therapy decisions could affect the clinical outcomes of subsequent IO therapies, although selection of the optimal second-line therapy will require further research.

Other studies have examined whether the second-line treatment outcomes will vary according to the duration of the previous therapy. ${ }^{12,15,17}$ A stratified analysis of the phase III trial INTORSECT (investigating Torisel as second-line therapy) data showed no significant PFS advantage for second-line sorafenib versus temsirolimus, regardless of the previous sunitinib therapy duration. ${ }^{15}$ The median survival was significantly longer for patients treated with second-line sorafenib versus temsirolimus for patients who had received first-line sunitinib treatment for $>180$ days but not for those with a treatment duration of $\leq 180$ days. ${ }^{15}$ The investigators proposed that this discrepancy between PFS and OS could relate to the unaccounted for use of poststudy anticancer therapy. ${ }^{15}$ Another study that showed a differential effect of second-line therapy according to the duration of first-line therapy was a phase III trial comparing sorafenib and axitinib. ${ }^{17}$ In the axitinib arm, patients with longer previous cytokine therapy had significant PFS and OS advantages. ${ }^{17}$ The same was true in the sorafenib arm, with patients with longer previous cytokine or sunitinib treatment experiencing better OS. Similarly, an analysis of the data from CheckMate 025, the phase III trial comparing second-line nivolumab to everolimus, found that for patients treated with second-line nivolumab, the OS for those with $\geq 6$ months versus $<6$ months of first-line therapy was 27.4 months (95\% CI, 23.2 months to not reached) versus 18.2 months (95\% CI, 13.9-25.0 months). ${ }^{12}$ The present study, which focused on real-world patients and has improved on previous analyses by adjusting for confounding, also observed numeric favorability in the TTD and TTNT for second-line IO therapy among patients with $\geq 6$ months of first-line sunitinib therapy versus $<6$ months and, thus, a significant OS benefit for those with $\geq 6$ months of first-line sunitinib therapy versus $<6$ months.

Biologic reasons exist for why a longer duration of first-line treatment might be associated with better OS after second-line treatment. ${ }^{18-21}$ The association might partly reflect the underlying tumor biology. Thus, patients with slower growing tumors might continue with the initial treatment longer and survive longer with 
Figure 3 Physician-assessed Best Response to Second-line Immuno-oncology (10) Therapy Stratified by Initial Best Response to First-line Sunitinib Therapy. ${ }^{1,2}$ 'Based on Patients' Best Response to First-line Sunitinib Therapy, Patients Were Grouped Into 3 Categories: CR1/PR1, SD1, and PD1. Among Those 3 Groups, the Patient's Best Response to Second-line I0 Therapy was Assessed and Presented Numerically and in bar Graph Form. ${ }^{2}$ Patients With Information Available on First- and Second-line Physician-assessed Best Response Were Included in the Analysis

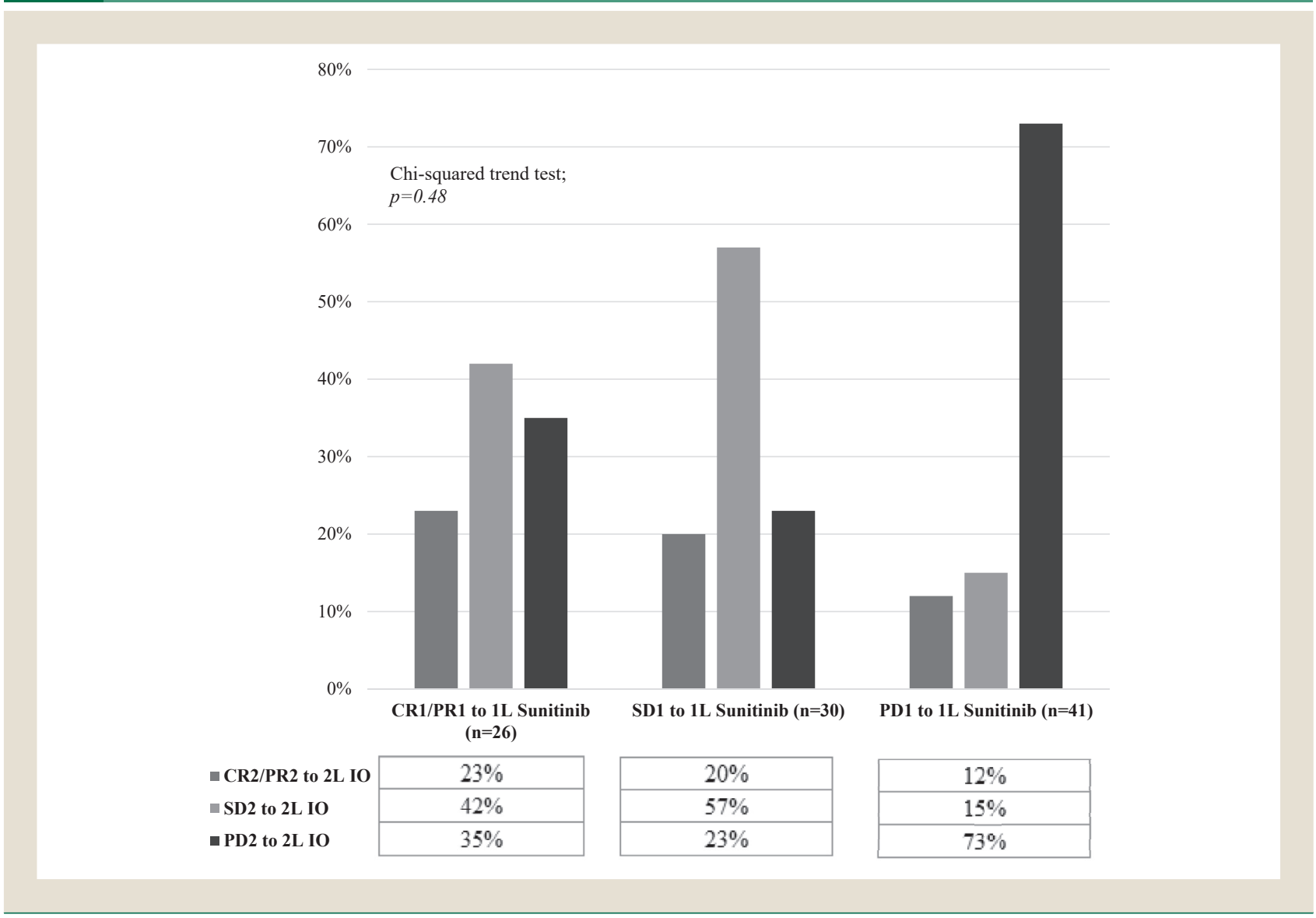

Abbreviations: $1 \mathrm{~L}=$ first line; $2 \mathrm{~L}=$ second line; $\mathrm{CR} 1=$ complete response to first line; $\mathrm{CR} 2=$ complete response to second line; PD1 = progressive disease with first line; PD2 = progressive disease with second line; PR1 = partial response to first line; PR2 = partial response to second line; SD1 = stable disease to first line; SD2 = stable disease to second line.

subsequent therapy. In contrast, patients with rapidly progressive disease and deterioration in IMDC risk factors will have shorter survival. This association could also vary according to the type of first-line treatment used and tumor heterogeneity. The results from previous studies have suggested that a patient's response to nivolumab might be influenced by earlier TKI treatment that affected the tumor microenvironment. ${ }^{11,12,22}$ Sunitinib has been shown to have direct antitumor effects and to exacerbate intratumoral heterogeneity, and checkpoint immunotherapies work best with high mutational burden tumors, although this is still being studied in mRCC. ${ }^{9,23,24}$

The treatment landscape for mRCC has changed from the treatment paradigm in which advanced RCC was treated with a VEGF inhibitor, followed by mammalian target of rapamycintargeted therapy. ${ }^{3}$ The approval of the combination of nivolumab plus ipilimumab for front-line treatment of patients with advanced RCC with an intermediate or a poor IMDC risk and avelumab plus axitinib or pembrolizumab plus axitinib for first-line treatment for all patients with mRCC have led to a changed treatment landscape. ${ }^{5,7,8}$ Thus, most of the patients in the present study might have been treated differently today. However, some patients will still initiate first-line sunitinib or TKI therapy for favorable-risk disease or various reasons, including the inability to use IO therapy because of autoimmune disease, steroid requirements, IO therapy costs, or access because many areas worldwide might not have IO therapy or combination therapies available. Therefore, the findings in the present study will be important for patient counseling.

The present study had some limitations. First, with any analysis of nonrandomized treatment groups, unmeasured confounding and potential biases (eg, selection bias) could account for the observed associations. For the TTNT, TTD, and physician-assessed best response, we attempted to reduce the bias by adjusting for confounders, including age, sex, and IMDC risk group. For OS, an IPW analysis was used such that exposure was not associated with time-varying covariates; thus, confounding by measured potential confounders was eliminated. Second, because the present study was from 2006 to 2018, the sunitinib treatment practices could have changed over time and affected the results. However, this effect was likely minimal because the sunitinib initiation year was not significantly different between those receiving $\geq 6$ months and those receiving $<6$ months of treatment (Table 1 ). Third, we had missing data, which could have biased the study results if the missing data 


\section{Sunitinib Followed by IO Checkpoint Inhibitors for mRCC}

were not completely random. Further analysis showed that only a small proportion of patients ( 25 of $161 ; 15 \%$ ) had missing data for the IMDC risk group. Thus, bias from missing data might have been minimal. Furthermore, in contrast to clinical trials with protocol-specified definitions of clinical events, the progression and clinical response assessments in retrospective studies of real-world clinical practice might not be performed consistently for all patients and across physician practices. Finally, the sample size of patients with $<6$ months of sunitinib treatment was limited.

\section{Conclusions}

The present study showed that the first-line sunitinib treatment duration might not be associated with TTNT, TTD, and tumor response with second-line IO therapy. This suggests that first-line sunitinib resistance will not preclude a second-line IO therapy response, and the mechanisms of resistance might not overlap. The present study also found that patients receiving IO therapy with firstline sunitinib treatment for $\geq 6$ months versus $<6$ months might have a survival advantage, although other unmeasured confounders might have been present. The findings from the present study might aid healthcare practitioners in deciding how to sequence therapies, which involves both the choice of therapy and its duration. The findings from the present study support the paradigm that previous therapy will not dictate the efficacy of subsequent IO therapy.

\section{Clinical Practice Points}

- Previous sunitinib therapy will not dictate the effectiveness of subsequent immunotherapy.

- We found no significant association between the first-line sunitinib duration and the clinical outcomes with second-line IO therapy.

- The findings from the present study have provided information on how the initial targeted therapy treatment decisions might affect the clinical outcomes of subsequent IO therapies, although the selection of the optimal second-line therapy is still warranted.

\section{Acknowledgments}

The present study was sponsored by Pfizer, Inc (New York, NY). The authors would like to thank Caroline Korves, ScD, and Rose Chang, MSPH, MS, ScD, of Analysis Group, Inc, for their assistance with developing our report.

\section{Disclosure}

J.C.W. has received travel support from Pfizer. B.B. has received research support from Pfizer and speaker fees from Ipsen, Amgen, and Pfizer. G.A.B. has received honoraria from Pfizer, Novartis, Bristol-Myers Squibb, Eisai, and Ipsen; research funding from Pfizer, Novartis, Bristol-Myers Squibb, Eisai, and Ipsen; and travel funding from Pfizer and Merck and has served as a consultant for Pfizer and Novartis. F.D. has received research support from Pfizer, Novartis, and Ipsen. A.H. has served on the advisory board for Pfizer, Roche, Merck, AstraZeneca, Ipsen, and Bristol-Myers Squibb; and research support from Genentech, Roche, Merck, GlaxoSmithKline, Bristol-Myers Squibb, and Novartis. R.R.M. has received research support from Pfizer and Bayer and served on the advisory board for Janssen and Novartis.
U.V. has received research support and honoraria from, and served as a consultant for, Pfizer, Bristol-Myers Squibb, Exelixis, and Bayer. G.D.V. served as a consultant or in an advisory role for Janssen,;Pfizer, Novartis, Bayer, Astellas Medivation, BristolMyers Squibb, and Pierre Fabre; research support from Ipsen, Pfizer, and Roche; has another relationship with Janssen; and has received funding from the Instituto de Salud Carlos III (grant PI17/01728). M.S.D., L.H., and C.N. are employees of Analysis Group, Inc, which received funding from Pfizer to conduct the present research study and develop the report. G.Z. and K.R. are employees of Pfizer. T.C. has received research support (institutional and personal) from AstraZeneca, Alexion, Bayer, Bristol Myers-Squibb/ER Squibb and Sons LLC, Cerulean, Eisai, Foundation Medicine Inc, Exelixis, Ipsen, Tracon, Genentech, Roche, Roche Products Limited, F. Hoffmann-La Roche, GlaxoSmithKline, Lilly, Merck, Novartis, Peloton, Pfizer, Prometheus Labs, Corvus, Calithera, Analysis Group, Sanofi/Aventis, and Takeda; honoraria from AstraZeneca, Alexion, Sanofi-Aventis, Bayer, Bristol Myers-Squibb/ER Squibb and Sons LLC, Cerulean, Eisai, Foundation Medicine Inc, Exelixis, Genentech, Roche, Roche Products Limited, F. Hoffmann-La Roche, GlaxoSmithKline, Merck, Novartis, Peloton, Pfizer, EMD Serono, Prometheus Labs, Corvus, Ipsen, Up-to-Date, NCCN, Analysis Group, NCCN, Michael J. Hennessy (MJH) Associates, Inc (Healthcare Communications Company, with several brands such as OnClive, PeerView, and PER), L-path, Kidney Cancer Journal, Clinical Care Options, Platform Q, Navinata Healthcare, Harborside Press, American Society of Medical Oncology, New England Journal of Medicine, Lancet Oncology, Heron Therapeutics, and Lilly; has had a consulting or advisory role for AstraZeneca, Alexion, Sanofi/Aventis, Bayer, Bristol Myers-Squibb/ER Squibb and Sons LLC, Cerulean, Eisai, Foundation Medicine Inc, Exelixis, Genentech, Heron Therapeutics, Lilly, Roche, GlaxoSmithKline, Merck, Novartis, Peloton, Pfizer, EMD Serono, Prometheus Labs, Corvus, Lilly, Ipsen, Up-to-Date, NCCN, and Analysis Group; and has received travel, accommodations, and expenses in relation to consulting, advisory roles, or honoraria. Medical writing and editorial assistance support could have been funded by communications companies funded by pharmaceutical companies. The institution (Dana-Farber Cancer Institute) could have received additional independent funding from drug companies and/or royalties potentially involved in research of the subject matter. D.H. has served as a consultant and received honoraria from Pfizer, Novartis, and Bristol-Myers Squibb. The remaining authors declare that they have no competing interests.

\section{Supplemental Data}

Supplemental tables accompanying this article can be found in the online version at https://doi.org/10.1016/j.clgc.2019.12.007.

\section{References}

1. Alsharedi M, Katz H. Check point inhibitors a new era in renal cell carcinoma treatment. Med Oncol 2018; 35:85.

2. Choueiri TK, Motzer RJ. Systemic therapy for metastatic renal-cell carcinoma. N Engl J Med 2017; 376:354-66.

3. Xu JX, Maher VE, Zhang L, et al. FDA approval summary: nivolumab in advanced renal cell carcinoma after anti-angiogenic therapy and exploratory predictive biomarker analysis. Oncologist 2017; 22:311-7. 
4. European Medicines Agency. Assessment report: OPDIVO international nonproprietary name: nivolumab. 2015. Available at: https://www.ema.europa.eu/ en/medicines/human/EPAR/opdivo. Accessed: January 30, 2019.

5. U.S. Food and Drug Administration. FDA approves nivolumab plus ipilimumab combination for intermediate or poor-risk advanced renal cell carcinoma. Available at: https://www.fda.gov/drugs/resources-information-approved-drugs/fdaapproves-nivolumab-plus-ipilimumab-combination-intermediate-or-poor-riskadvanced-renal-cell. Accessed: August 21, 2018.

6. European Medicines Agency. Positive Opinion on the Change to the Marketing Authorisation for Opdivo (nivolumab) and Yervoy (ipilimumab). 2018. Available at: https:/www.ema.europa.eu/en/documents/smop/questions-answers-positiveopinion-change-marketing-authorisation-opdivo-nivolumab-yervoy-ipilimumab_ en.pdf. Accessed: January 30, 2019.

7. Rini BI, Plimack ER, Stus V, et al. Pembrolizumab plus axitinib versus sunitinib for advanced renal-cell carcinoma. N Engl J Med 2019; 380:1116-27.

8. Motzer RJ, Penkov K, Haanen J, et al. Avelumab plus axitinib versus sunitinib for advanced renal-cell carcinoma. N Engl J Med 2019; 380:1103-15.

9. Huillard O, Alexandre J, Goldwasser F. Treatment of advanced renal-cell carcinoma. $N$ Engl J Med 2016; 374:888.

10. Le DT, Uram JN, Wang H, et al. PD-1 blockade in tumors with mismatch-repair deficiency. N Engl J Med 2015; 372:2509-20.

11. Stewart GD, O'Mahony FC, Laird A, et al. Sunitinib treatment exacerbates intratumoral heterogeneity in metastatic renal cancer. Clin Cancer Res 2015; 21:4212-23.

12. Escudier B, Sharma P, McDermott DF, et al. CheckMate 025 randomized phase 3 study: outcomes by key baseline factors and prior therapy for nivolumab versus everolimus in advanced renal cell carcinoma. Eur Urol 2017; 72:962-71.

13. Heng DY, Xie W, Regan MM, et al. External validation and comparison with other models of the International Metastatic Renal-Cell Carcinoma Database Consortium prognostic model: a population-based study. Lancet Oncol 2013; 14:141-8.

14. US Department of Health and Human Services FDA, Center for Drug Evaluation and Research, Center for Biologics Evaluation and Research. Guidance for Industry: Clinical Trial Endpoints for the Approval of Cancer Drugs and Biologics.
2007. Available at: https://www.fda.gov/regulatory-information/search-fdaguidance-documents/clinical-trial-endpoints-approval-cancer-drugs-and-biologics. Accessed: January 30, 2019.

15. Hutson TE, Escudier B, Esteban E, et al. Randomized phase III trial of temsirolimus versus sorafenib as second-line therapy after sunitinib in patients with metastatic renal cell carcinoma. J Clin Oncol 2014; 32:760-7.

16. D’Agostino RB, Lee ML, Belanger AJ, Cupples LA, Anderson K, Kannel WB. Relation of pooled logistic regression to time dependent Cox regression analysis: the Framingham Heart Study. Stat Med 1990; 9:1501-15.

17. Escudier B, Michaelson MD, Motzer RJ, et al. Axitinib versus sorafenib in advanced renal cell carcinoma: subanalyses by prior therapy from a randomised phase III trial. Br I Cancer 2014; 110:2821-8.

18. Verbiest A, Renders I, Caruso S, et al. Clear-cell renal cell carcinoma: molecular characterization of IMDC risk groups and sarcomatoid tumors. Clin Genitourin Cancer 2019; 17:e981-94.

19. Beuselinck B, Verbiest A, Couchy G, et al. Pro-angiogenic gene expression is associated with better outcome on sunitinib in metastatic clear-cell renal cell carcinoma. Acta Oncol 2018; 57:498-508.

20. Beuselinck B, Job S, Becht E, et al. Molecular subtypes of clear cell renal cell carcinoma are associated with sunitinib response in the metastatic setting. Clin Cancer Res 2015; 21:1329-39.

21. McDermott DF, Huseni MA, Atkins MB, et al. Clinical activity and molecular correlates of response to atezolizumab alone or in combination with bevacizumab versus sunitinib in renal cell carcinoma. Nat Med 2018; 24:749-57.

22. Flippot R, Escudier B, Albiges L. Immune checkpoint inhibitors: toward new paradigms in renal cell carcinoma. Drugs 2018; 78:1443-57.

23. Miao D, Margolis CA, Gao W, et al. Genomic correlates of response to immune checkpoint therapies in clear cell renal cell carcinoma. Science 2018; 359:801-6.

24. Yarchoan M, Hopkins A, Jaffee EM. Tumor mutational burden and response rate to PD-1 inhibition. N Engl J Med 2017; 377:2500-1. 


\section{Sunitinib Followed by IO Checkpoint Inhibitors for mRCC}

\section{Supplemental Data}

\section{Supplemental Table 1 Treatment Sequence of Patients Stratified by First-line Sunitinib Before Second-line 10 Therapy}

\begin{tabular}{|c|c|c|c|}
\hline \multirow[b]{2}{*}{ Characteristic } & \multicolumn{2}{|c|}{ First-line Sunitinib Duration, n (\%) } & \multirow[b]{2}{*}{$P$ Value } \\
\hline & $<6 \mathrm{mo}(\mathrm{n}=45)$ & $\geq 6 \mathrm{mo}(\mathrm{n}=116)$ & \\
\hline Second-line treatment & 45 & 116 & \\
\hline \multicolumn{4}{|l|}{10 therapy } \\
\hline Nivolumab $^{\mathrm{a}}$ & $44(97.8)$ & $110(94.8)$ & .67 \\
\hline Atezolizumab $^{\mathrm{b}}$ & $1(2.2)$ & $2(1.7)$ & $>.99$ \\
\hline Avelumab $^{\mathrm{C}}$ & $0(0.0)$ & $2(1.7)$ & $>.99$ \\
\hline Pembrolizumab $^{d}$ & $0(0.0)$ & $2(1.7)$ & $>.99$ \\
\hline Third-line treatment & $15(33.3)$ & $45(38.8)$ & .52 \\
\hline \multicolumn{4}{|l|}{10 therapy } \\
\hline Nivolumab ${ }^{\mathrm{e}}$ & $1(6.7)$ & $2(4.4)$ & $>.99$ \\
\hline Atezolizumab $^{b}$ & $1(6.7)$ & $0(0.0)$ & .25 \\
\hline \multicolumn{4}{|l|}{ Targeted therapy } \\
\hline Axitinib & $5(33.3)$ & $18(40.0)$ & .65 \\
\hline Everolimus & $0(0.0)$ & $9(20.0)$ & .10 \\
\hline Cabozantinib & $2(13.3)$ & $6(13.3)$ & $>.99$ \\
\hline Investigational drug & $1(6.7)$ & $4(8.9)$ & $>.99$ \\
\hline Sorafenib & $1(6.7)$ & $3(6.7)$ & $>.99$ \\
\hline Pazopanib & $2(13.3)$ & $2(4.4)$ & .26 \\
\hline Sunitinib & $1(6.7)$ & $1(2.2)$ & .44 \\
\hline Temsirolimus & $1(6.7)$ & $0(0.0)$ & .25 \\
\hline
\end{tabular}

Abbreviation: 10 = immuno-oncology

${ }^{a}$ Nivolumab in second-line therapy included nivolumab treatment alone $(n=148)$ and combined with other therapy $(n=6)$.

${ }^{\mathrm{b}}$ Atezolizumab was combined with other treatment.

${ }^{\mathrm{c} A v e l u m a b}$ in second-line therapy included avelumab treatment alone $(n=1)$ and combined with other therapy $(n=1)$.

${ }^{d}$ Pembrolizumab in second-line therapy included pembrolizumab treatment alone $(n=1)$ and combined with other therapy $(n=1)$.

${ }^{e}$ Nivolumab in third-line therapy included nivolumab treatment alone $(n=1)$ and combined with other therapy $(n=2)$.

\section{Supplemental Table 2 Physician-assessed Best Response Stratified by First-line Sunitinib Duration Before Second-line 10 Therapy}

\begin{tabular}{|c|c|c|c|}
\hline \multirow{2}{*}{$\begin{array}{l}\text { Physician-assessed Best } \\
\text { Response }\end{array}$} & \multicolumn{2}{|c|}{ First-line Sunitinib Duration, n (\%) } & \multirow[b]{2}{*}{$P$ Value } \\
\hline & $<6 \mathrm{mo}(\mathrm{n}=45)$ & $\geq 6 \mathrm{mo}(\mathrm{n}=116)$ & \\
\hline \multicolumn{4}{|l|}{ First-line treatment (sunitinib) } \\
\hline Complete/partial response (ORR) & $2(6.3)$ & $31(30.1)$ & $.01^{\mathrm{a}}$ \\
\hline Complete response & $0(0.0)$ & $3(2.9)$ & NA \\
\hline Partial response & $2(6.3)$ & $28(27.2)$ & $.01^{\mathrm{a}}$ \\
\hline Stable disease & $7(21.9)$ & $38(36.9)$ & .12 \\
\hline Progressive disease & $23(71.9)$ & $34(33.0)$ & $.0001^{\mathrm{a}}$ \\
\hline \multicolumn{4}{|l|}{ Second-line treatment (10) } \\
\hline Complete/partial response (ORR) & $6(23.1)$ & $15(18.5)$ & .61 \\
\hline Complete response & $0(0.0)$ & $3(3.7)$ & NA \\
\hline Partial response & $6(23.1)$ & $12(14.8)$ & .37 \\
\hline Stable disease & $8(30.8)$ & $27(33.3)$ & .81 \\
\hline Progressive disease & $12(46.2)$ & $39(48.1)$ & .86 \\
\hline
\end{tabular}

Abbreviation: 10 = immuno-oncology; $\mathrm{NA}=$ not applicable; $\mathrm{ORR}=$ overall response rate. ${ }^{a} p<.05$. 